\title{
Cystic fibrosis transmembrane conductance regulator dysfunction in platelets drives lung hyperinflammation
}

\author{
Guadalupe Ortiz-Muñoz, ${ }^{1}$ Michelle A. Yu, ${ }^{1}$ Emma Lefrançais, ${ }^{1}$ Beñat Mallavia, ${ }^{1}$ Colin Valet, ${ }^{1}$ Jennifer J. Tian, ${ }^{1}$ Serena Ranucci, ${ }^{1}$ \\ Kristin M. Wang, ${ }^{1}$ Zhe Liu, ${ }^{1}$ Nicholas Kwaan, ${ }^{1}$ Diana Dawson, ${ }^{1}$ Mary Ellen Kleinhenz, ${ }^{1}$ Fadi T. Khasawneh, ${ }^{2}$ Peter M. Haggie, ${ }^{1,3}$ \\ Alan S. Verkman, ${ }^{1,3}$ and Mark R. Looney ${ }^{1,4}$ \\ 1Department of Medicine, UCSF, San Francisco, California, USA. ${ }^{2}$ School of Pharmacy, University of Texas, El Paso, El Paso, Texas, USA. ${ }^{3}$ Department of Physiology and ${ }^{4}$ Department of Laboratory Medicine, \\ UCSF, San Francisco, California, USA.
}

Cystic fibrosis (CF) lung disease is characterized by an inflammatory response that can lead to terminal respiratory failure. The cystic fibrosis transmembrane conductance regulator (CFTR) is mutated in CF, and we hypothesized that dysfunctional CFTR in platelets, which are key participants in immune responses, is a central determinant of CF inflammation. We found that deletion of CFTR in platelets produced exaggerated acute lung inflammation and platelet activation after intratracheal LPS or Pseudomonas aeruginosa challenge. CFTR loss of function in mouse or human platelets resulted in agonist-induced hyperactivation and increased calcium entry into platelets. Inhibition of the transient receptor potential cation channel 6 (TRPC6) reduced platelet activation and calcium flux, and reduced lung injury in CF mice after intratracheal LPS or Pseudomonas aeruginosa challenge. CF subjects receiving CFTR modulator therapy showed partial restoration of CFTR function in platelets, which may be a convenient approach to monitoring biological responses to CFTR modulators. We conclude that CFTR dysfunction in platelets produces aberrant TRPC6-dependent platelet activation, which is a major driver of CF lung inflammation and impaired bacterial clearance. Platelets and TRPC6 are what we believe to be novel therapeutic targets in the treatment of CF lung disease.

\section{Introduction}

Cystic fibrosis (CF) is an autosomal recessive disease caused by mutations in the cystic fibrosis transmembrane conductance regulator (CFTR) that affects 30,000 people in the United States $(1,2)$. Progressive lung inflammation and impaired bacterial clearance can lead to terminal respiratory failure, which accounts for $85 \%$ of all CF mortalities. Despite major advances that have improved the life expectancy for patients with CF, including cloning CFTR in 1989 (3), recombinant human DNAse in 1994 (4), and CFTR modulators to restore partial function of mutant channels (5-8) in the last decade, modern therapy has only extended the median age at death to 30 years, which lags far behind the median life expectancy for Americans who do not have CF $(1,9)$. As more patients with $\mathrm{CF}$ reach adulthood, chronic inflammation results in irreversible injury to multiple organs.

The majority of CF investigations have focused on mechanisms by which impaired or absent CFTR function in the surface airway epithelium leads to mucus impaction, inflammation, and bronchiectasis. This prevailing theory suggests that viscous secretions, decreased airway surface liquid volume and $\mathrm{pH}$, and

\section{Related Commentary: p. 1632}

Authorship note: GOM and MAY are co-first authors.

Conflict of interest: The authors have declared that no conflict of interest exists. Copyright: @ 2020, American Society for Clinical Investigation.

Submitted: April 17, 2019; Accepted: January 14, 2020; Published: March 16, 2020 Reference information: / Clin Invest. 2020;130(4):2041-2053.

https://doi.org/10.1172/JCI129635. dysfunctional mucus clearance lead to chronic lung infection and inflammation (10). An alternative paradigm has emerged in which CFTR dysfunction produces a chronic hyperinflammatory state that precedes infection, drives structural disease, and impairs bacterial clearance. Supporting this theory, bronchiectasis has been demonstrated in infants as early as 3 months of age - a time point that precedes recurrent infections and mucus impaction (11). In ferret models of $\mathrm{CF}$, there is evidence of early hyperinflammation in the absence of infection $(12,13)$. Although controversial in CF pig models $(14,15)$, this theory is also supported by evidence that mice with the most common CF mutation, $\triangle F 508$, exhibit increased lung inflammation and injury after LPS challenge (16), supporting an abnormal inflammatory response in CF. However, the mechanisms driving hyperinflammation in CF remain unknown.

CFTR is expressed in many different cell types, including hematopoietic cells (17-21). An exciting discovery was the transplantation of bone marrow from WT mice into $\mathrm{CFTR}^{-/-}$mice that corrected the hyperinflammatory cytokine response observed after lung injury with LPS (17). This work implicated macrophages as a major contributor to the abnormal CF lung inflammatory response (22), yet other inflammatory cells may play important roles in this process.

Platelets have emerged as key mediators of inflammation and injury in a wide variety of disease states (23). Platelet depletion or antiplatelet therapies attenuate injury and mortality in animal models of sterile acute lung injury $(24,25)$, but it is clear that platelets are required for host defense during bacterial pneumonia (2628). CFTR expression has been demonstrated on human platelets through functional and biochemical approaches (21). Patch-clamp characterization, albeit challenging in platelets, demonstrates 

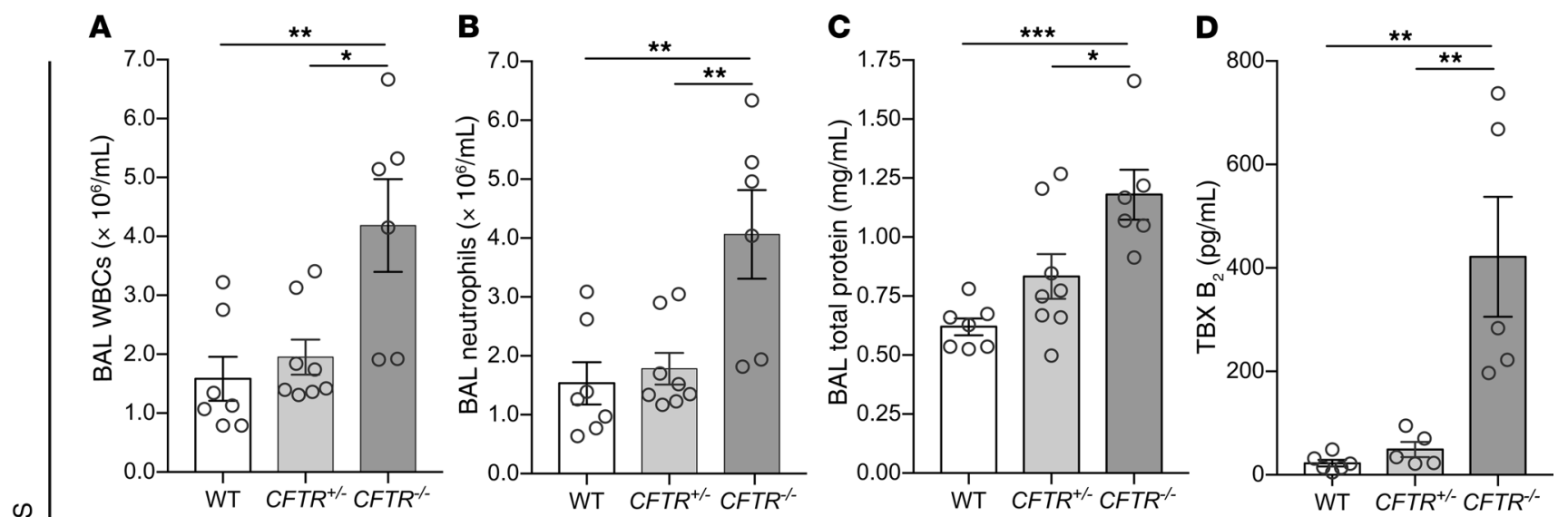

D
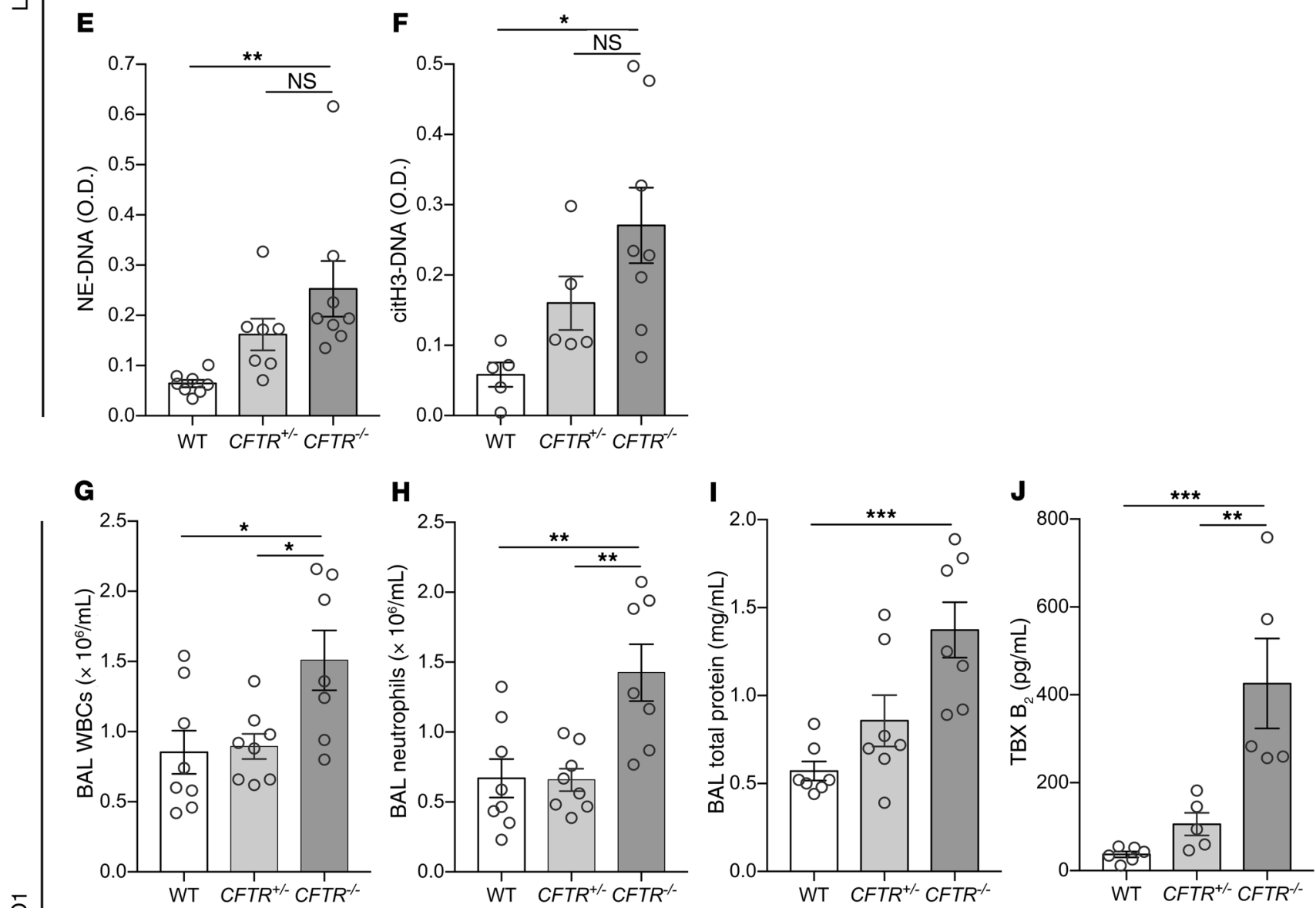

옴
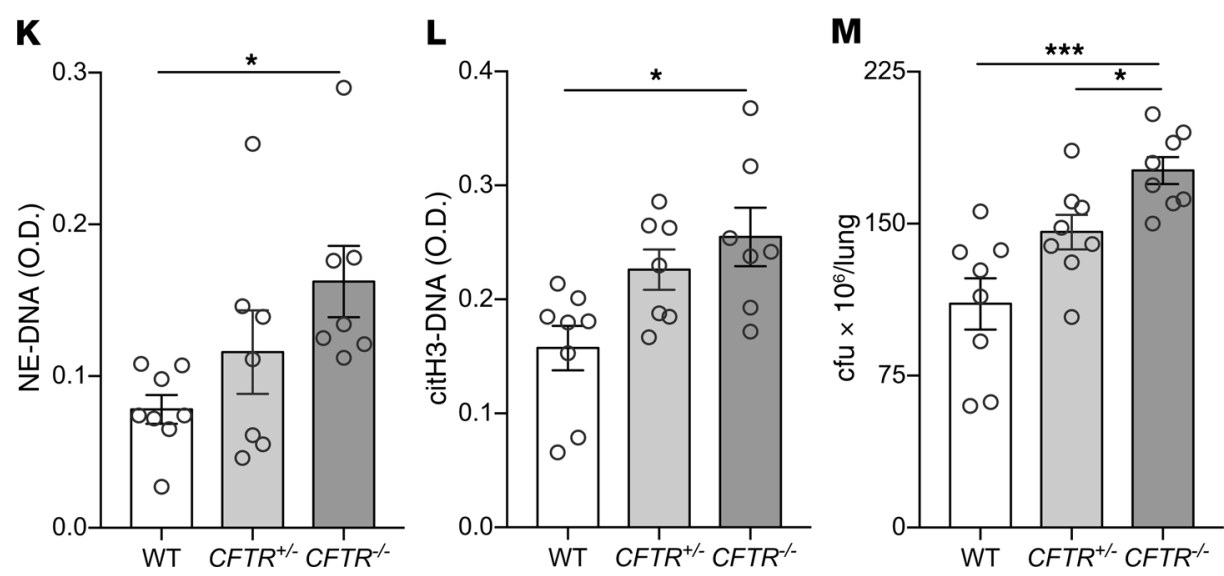
Figure 1. Lung injury and bacterial lung colony measurements in CF mice after intratracheal LPS or PA01. (A and G) BAL WBCs, (B and H) BAL neutrophils, ( $\mathbf{C}$ and $\mathbf{I}) \mathrm{BAL}$ total protein, ( $\mathbf{D}$ and $\mathbf{J}) \mathrm{BAL}$ thromboxane $\mathrm{B} 2$, and BAL NETs as measured by ( $\mathbf{E}$ and $\mathbf{K})$ NE-DNA and ( $\mathbf{F}$ and $\mathbf{L}$ ) citH3-DNA in WT, $\mathrm{CFTR}^{+/-}$, and $\mathrm{CFTR}^{-/-}$mice at 48 hours after intratracheal LPS or PA01. (M) Bacterial lung colony counts in WT, $\mathrm{CFTR}^{+/-}$, and $\mathrm{CFTR}^{-/-}$mice after intratracheal PA01. Data are mean \pm SEM of 5 to 8 animals per group. Data were analyzed by 1-way ANOVA. ${ }^{*} P \leq 0.05 ;{ }^{*} P \leq 0.01 ;{ }^{* *} P \leq 0.001$.

platelet chloride channels that regulate internal calcium and thereby activation (29). Importantly, CF human subjects have increased circulating activated platelets and leukocyte-platelet aggregates (30). Treatment with ibuprofen slowed the progression of CF lung disease (31), and ibuprofen has been shown to decrease thromboxane generation during acute inflammation in humans (32), which suggests that platelet activation is a targetable mediator of CF lung disease. Anti-platelet therapy in the $\triangle \mathrm{F} 508$ mouse model reduces lung injury (33). Taken together, these observations support a potentially important role of CFTR in platelets in regulating lung inflammation in CF.

Using lineage-specific deletion of CFTR in models of acute lung inflammation, we tested the role of CFTR in immune cells in contributing to hyperinflammation. We hypothesized that CFTR dysfunction in platelets produces aberrant calcium entry, leading to platelet activation and hyperinflammation. We tested the relationship between CFTR and transient receptor potential cation channel 6 (TRPC6), a calcium channel that demonstrates reciprocal functional interaction with CFTR in bronchial epithelial cells (34) and has been shown to regulate platelet calcium homeostasis $(35,36)$.

\section{Results}

$\mathrm{CFTR}^{-/}$mice exhibit increased lung injury, neutrophil extracellular traps (NETs), and platelet activation after LPS and PAO1 challenge. To investigate the effect of global deletion of CFTR on lung inflammation, we challenged $\mathrm{CFTR}^{-/-}$mice with intratracheal LPS or Pseudomonas aeruginosa (PAO1). At 48 hours after instillation, CFTR ${ }^{-1}$ mice showed increased bronchoalveolar lavage (BAL) white blood cell (WBC) and neutrophil counts compared with WT or $\mathrm{CFTR}^{+/}$ mice in the LPS (Figure 1, A and B) and PAO1 models (Figure 1, G and $\mathrm{H}$ ). Global CFTR deletion increased lung permeability as determined by cell-free BAL total protein, whereas $\mathrm{CFTR}^{+/-}$and WT mice had similar protein leak in the LPS (Figure 1C) and PAO1 (Figure 1I) models. We tested for platelet activation by measuring BAL thromboxane $\mathrm{B}_{2}\left(\mathrm{TXB}_{2}\right)(24)$, and $C F T R^{-/-}$mice showed increased platelet activation (Figure 1, D and J) in both models. Since activated platelets can trigger NET formation (37-39), we tested for NETs, including citrullinated NETs, which were increased in the $\mathrm{CFTR}^{-/-}$BAL (Figure 1, E, F, K, and L) in both models. In the PAO1 model, $\mathrm{CFTR}^{--}$mice had increased BAL bacterial colony counts compared with WT and $\mathrm{CFTR}^{+/-}$mice (Figure $1 \mathrm{M}$ ).

Deletion of CFTR in hematopoietic cells. We confirmed a previous report (21) that CFTR is expressed on human platelets (Supplemental Figure 1A; supplemental material available online with this article; https://doi.org/10.1172/JCI129635DS1), but CFTR expression on mouse platelets has not been verified. We isolated platelets from WT and $\mathrm{CFTR}^{-/-}$mice and tested for expression using immunofluorescence. CFTR was detected on WT platelets $\left(\mathrm{CD}_{4} 1^{+}\right)$but not on $\mathrm{CFTR}^{-/-}$platelets (Supplemental Figure $1 \mathrm{~B})$. We used the $C F T R^{f / / f l}$ mouse (hereafter $\left.C F^{\mathrm{fl} / \mathrm{fl}}\right)(40)$ to generate lineage-specific deletion of CFTR in platelets (PF4-Cre $\mathrm{x}$ $\mathrm{CF}^{\mathrm{f} / \mathrm{fl}}$, hereafter CF-PF4) (41), myeloid cells (LysM-Cre x CF ${ }^{\mathrm{f} / \mathrm{fl}}$, hereafter CF-LysM) (42), and neutrophils (MRP8-Cre x CF ${ }^{\mathrm{f} / \mathrm{fl}}$, hereafter CF-MRP8) (43). To test for CFTR deletion efficiency, we isolated megakaryocytes from $\mathrm{CF}^{\mathrm{f} / \mathrm{fl}}$ and $\mathrm{CF}-\mathrm{PF} 4$ mice and neutrophils from $\mathrm{CF}^{\mathrm{t} / \mathrm{fl}}$ and CF-LysM mice. We detected CFTR mRNA in $C F^{\mathrm{l} / \mathrm{fl}}$ neutrophils and megakaryocytes, although the relative abundance of CFTR mRNA is much lower than in lung epithelial cells (Supplemental Table 1). CFTR mRNA was significantly reduced in CF-PF4 megakaryocytes and CF-LysM neutrophils compared with $\mathrm{CF}^{\mathrm{fl} / \mathrm{fl}}$ controls (Supplemental Table 1). We concluded that hematopoietic cell expression of CFTR could be effectively modulated for our experiments.

Lineage-specific CFTR deletion in platelets results in increased LPS-induced lung injury. At 48 hours after LPS challenge, plateletspecific CFTR deletion (CF-PF4) increased lung inflammation with increased BAL WBC and neutrophil counts compared with $\mathrm{CF}^{\mathrm{f} / \mathrm{fl}}$ controls, CF-LysM, and CF-MRP8 mice (Figure 2, A and B). $\mathrm{CF}-\mathrm{PF} 4$ mice had in increased lung permeability compared with $\mathrm{CF}^{\mathrm{f} / \mathrm{fl}}, \mathrm{CF}-\mathrm{LysM}$, or CF-MRP8 mice (Figure 2C). We have previously shown that after LPS challenge, platelets are retained in pulmonary capillaries and alveoli and form leukocyte-platelet aggregates (LPAs) that contribute to lung inflammation (25). We investigated the effect of CFTR deletion on heterotypic aggregate formation by quantifying LPAs 24 hours after LPS challenge. All mouse lines had increased neutrophil-platelet aggregates (NPAs; BAL and blood) when exposed to LPS compared with PBS controls, but the CF-PF4 line had the highest levels of BAL and blood NPAs (Figure 2, D and E). Blood monocyte-platelet aggregates (MPAs) were also highest in the CF-PF4 mice challenged with LPS (Figure $2 \mathrm{~F}$ ).

Platelet-specific CFTR deletion results in increased lung injury after bacterial challenge. With the striking inflammatory phenotype of the CF-PF4 mice established after LPS challenge, we next tested these mice in the PAO1 model. Compared with $\mathrm{CF}^{\mathrm{f} / \mathrm{l}}$ mice, CF-PF4 mice had increased BAL WBC and neutrophil counts (Figure 2, G and $\mathrm{H}$ ) and increased lung permeability (Figure 2I) at 48 hours after PAO1 challenge. CF-PF4 mice also had increased in vivo platelet activation, including a measurement of platelet degranulation (platelet surface CD62P), compared with $\mathrm{CF}^{\mathrm{fl} / \mathrm{fl}}$ mice (Figure 2, J-M). Similar to results obtained with the PAO1 model in $\mathrm{CFTR}^{-/-}$mice (Figure 1), CF-PF 4 mice ineffectively cleared bacteria compared with controls (Figure $2 \mathrm{~N}$ ).

CFTR inhibition or deletion leads to increased agonist-induced platelet activation. To characterize the effect of CFTR dysfunction on platelet activation, we used a flow cytometry assay for platelet $P$ selectin (CD62P) surface expression under thrombin-stimulated conditions. WT platelets were incubated with the CFTR inhibitor $\mathrm{CFTR}_{\text {inh }}-172$ (44). A representative gating scheme is shown with $\mathrm{CD} 41^{+} / \mathrm{CD} 2 \mathrm{P}^{+}$cells representing activated platelets (Figure $3 \mathrm{~A})$. With thrombin stimulation, $\mathrm{CFTR}^{-/-}$and $\mathrm{CF}-\mathrm{PF} 4$ platelets expressed increased CD62P compared with WT, $C F T R^{+/-}$, or $\mathrm{CF}^{\mathrm{f} / \mathrm{fl}}$ platelets, which was phenocopied by incubating $\mathrm{WT}$ or $\mathrm{CF}^{\mathrm{fl} / \mathrm{fl}}$ platelets with $\mathrm{CFTR}_{\text {inh }}-172$ (Figure 3B). CFTR ${ }^{+/}$platelets responded similarly to WT platelets in this assay (data not shown). To rule 

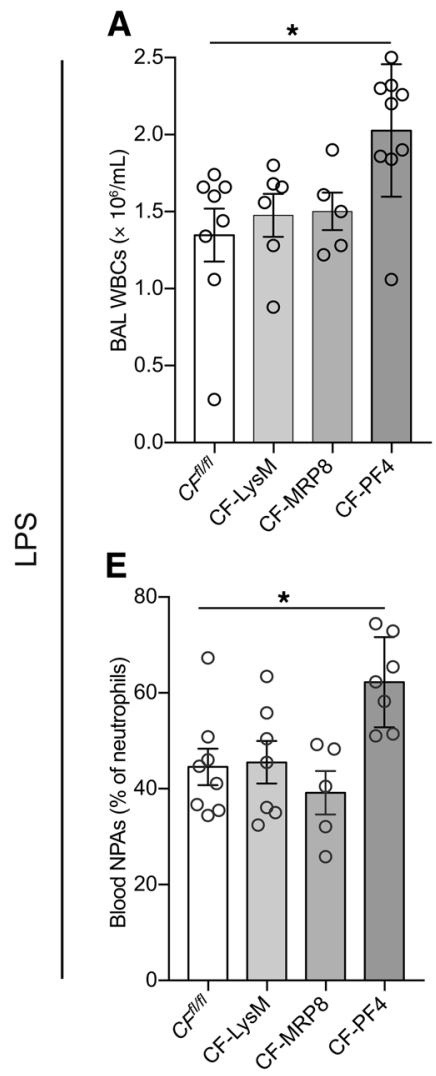

G

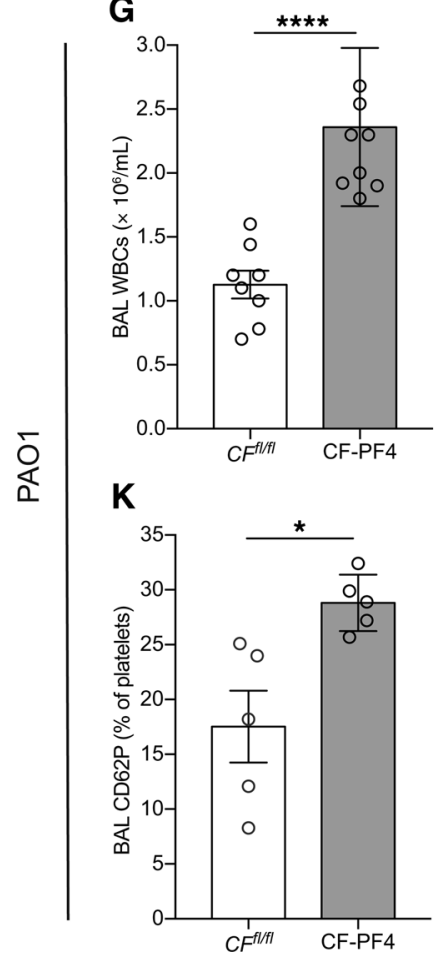

H

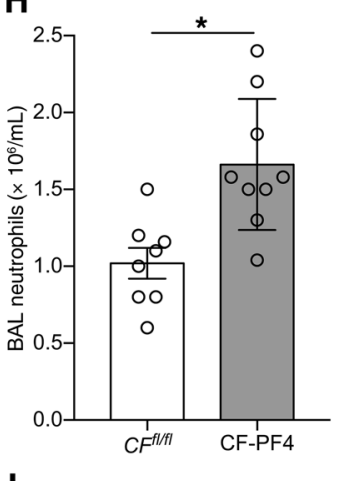

L

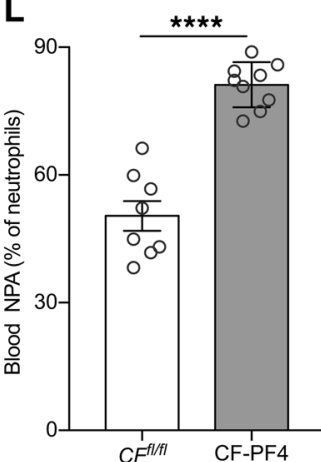

B
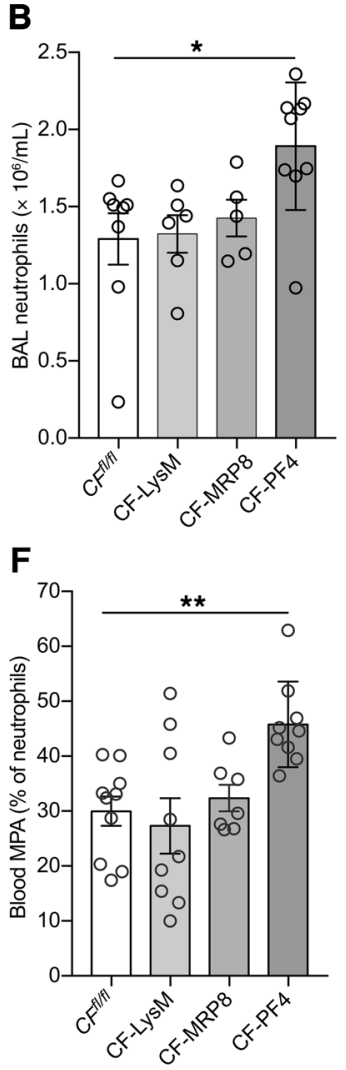

C

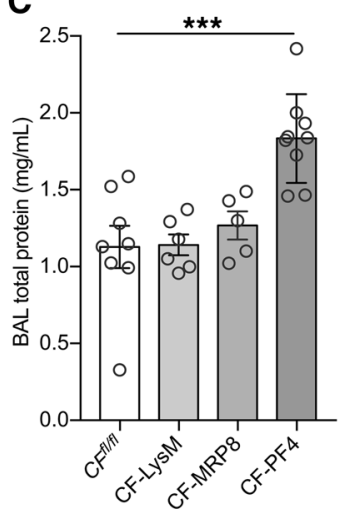

D

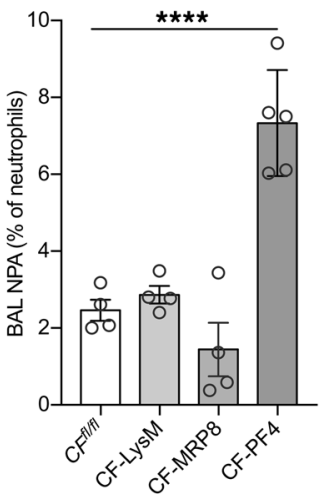

I
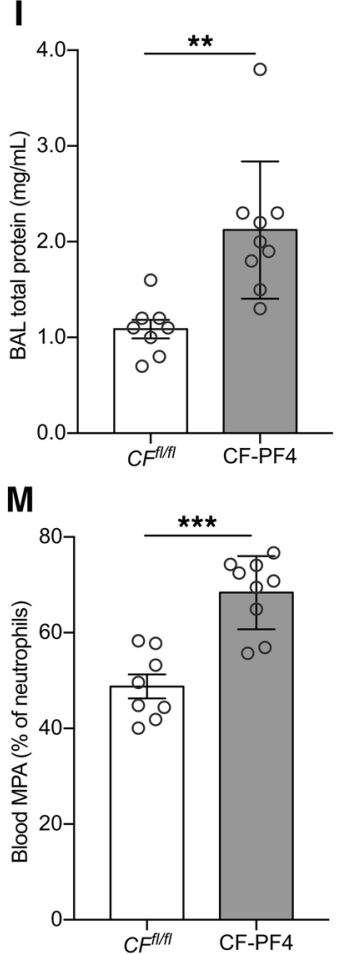

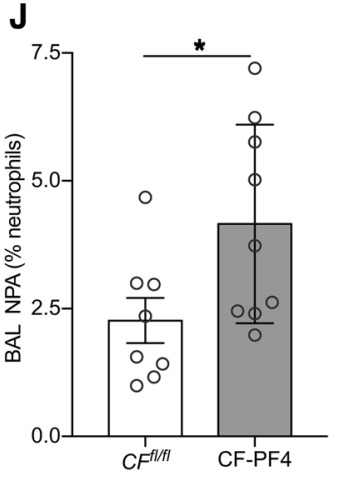

N

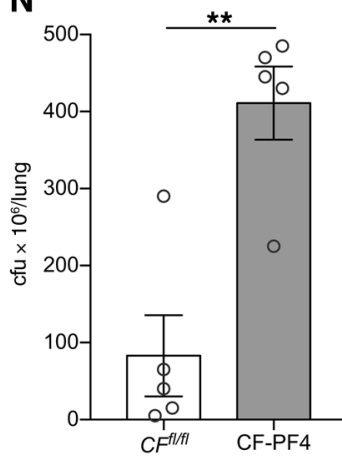

Figure 2. Lung injury and leukocyte-platelet aggregates in mice with conditional CFTR deletion after intratracheal LPS or PA01. (A) BAL WBCs, (B) neutrophils, (C) total protein in CF/lff, CF-LysM, CF-MRP8, and CF-PF4 mice at 48 hours after intratracheal LPS. NPAs in BAL (D) and blood (E), and MPAs in blood (F) at 24 hours after intratracheal LPS. Data are mean \pm SEM of 4 to 9 animals per group. Data were analyzed by 1-way ANOVA. (G-N) Lung injury and leukocyte-platelet aggregates in CFI/fl and CF-PF4 mice after intratracheal PA01. (G) BAL WBC, (H) neutrophils, (I) total protein, (J) NPAs, (K) CD62P, (L) blood NPAs, (M) blood MPAs, and (N) lung colonies in CF-PF4 and CF $F^{f / f l}$ mice after challenge with PAO1. Standard lung injury measurements were performed at 48 hours, and NPAs and MPAs at 24 hours after intratracheal PA01. Data are mean \pm SEM of 5 to 8 animals per group. Data were analyzed by Student's $t$ test. ${ }^{*} P \leq 0.05 ;{ }^{* *} P \leq 0.01 ;{ }^{* * *} P \leq 0.001 ;{ }^{* * *} P \leq 0.0001$. 
out an off-target effect of $\mathrm{CFTR}_{\text {inh }}-172$, we tested an unrelated CFTR inhibitor, BPO-27 (45), on WT platelets and also observed increased platelet activation (Supplemental Figure 2). Notably, there was no effect of $\mathrm{CFTR}_{\mathrm{inh}}-172$ on either $\mathrm{CFTR}^{-/-}$or CF-PF4 platelet activation (Figure $3 \mathrm{~B}$ ), which supports efficient deletion of CFTR from platelets. We tested for effects on platelet aggregation and tail bleeding in the $\mathrm{CF}^{\mathrm{fl} / \mathrm{fl}}$ and CF-PF4 mice, but found no differences (Supplemental Figure 3, A-D).

$C F$ platelet activation is reversed by TRPC6 inhibition. We next reasoned that since CFTR and TRPC channels are reciprocally coupled in epithelial cells (34), this relationship could also exist in platelets and underlie the activation phenotype in CF platelets. First, TRPC1-7 isoforms were analyzed by reverse transcriptionquantitative PCR (RT-qPCR) in isolated mouse platelets. Only TRPC1 and TRPC6 mRNA were detected in platelets, with minimal differences in expression of either isoform in platelets isolated from WT, CFTR ${ }^{-/}, \mathrm{CF}^{\mathrm{fl} / \mathrm{fl}}, \mathrm{CF}-\mathrm{LysM}$, or CF-PF4 strains (Figure 4, A and B). We focused on TRPC6 since it localizes to the plasma membrane of platelets whereas TRPC1 localizes to intracellular membranes (46), and the genetic deletion of TRPC1 in platelets does not result in altered calcium homeostasis or platelet function (47). We demonstrated TRPC6 expression on platelets isolated from WT but not $\mathrm{TRPC6}^{-/}$mice using immunofluorescence and flow cytometry (Figure 4, C and D). Pharmacologic inhibition of TRPC6 with SKF-96365 (SK), a TRPC6/3 inhibitor (48), reversed the increased CD62P expression in platelets with dysfunctional CFTR channels, including WT or $\mathrm{CF}^{\mathrm{fl} / \mathrm{fl}}$ platelets incubated with $\mathrm{CFTR}_{\text {inh }}-172, \mathrm{CFTR}^{-/}$platelets, and CF-PF4 platelets (Figure $4, \mathrm{E}$ and $\mathrm{F}$ ). Incubation with SK alone was no different than the CFTR $_{\text {inh }}-172+$ SK group (data not shown). The increased CD62P expression in $\mathrm{CFTR}^{-/-}$platelets was not observed in platelets isolated from $\mathrm{TRPC6}^{-/-}$or $\mathrm{CFTR}^{-/-} / \mathrm{TRPC6}^{-/-}$mice (Figure $4 \mathrm{E}$ ).

Platelets with deleted CFTR have increased calcium entry that is regulated by TRPC6. We hypothesized that TRPC6 activation in platelets results in increased calcium entry and activation. To test the effect of CFTR or TRPC6 inhibition on calcium entry, platelets were isolated and tested in a ratiometric Indo- 1 assay, during which thrombin is serially added at 60-second intervals (Figure 5A). Platelets from $\mathrm{CFTR}^{-/}$mice showed increased calcium entry as measured by Indo-1 peak median fluorescent intensity (MFI) compared with WT or TRPC6 ${ }^{-1}$ mice, which was attenuated by pharmacologic inhibition of TRPC6 (Figure 5B). WT platelets incubated with $\mathrm{CFTR}_{\text {inh }}-172$ showed increased calcium entry compared with vehicle, also rescued by TRPC6 inhibition (Figure 5B). Finally, $\mathrm{TRPC}^{-/}$platelets showed decreased calcium entry compared with $\mathrm{CFTR}^{-/-}$or WT mice and were unaffected by CFTR $_{\text {inh }}-172$ (Figure 5B). Incubation with SK alone was no different than the CFTR $_{\text {inh }}-172$ + SK group (data not shown).

TRPC6 inhibition normalizes calcium entry and platelet activation in human platelets with dysfunctional CFTR. We next tested the effects of CFTR or TRPC6 inhibition in platelets isolated from healthy controls or from $\mathrm{CF}$ subjects recruited during outpatient well-visits to the UCSF Adult CF Clinic (Supplemental Table 2). A proportion of the CF subjects, all $\Delta \mathrm{F} 508 / \Delta \mathrm{F} 508$, were treated with lumacaftor/ivacaftor (Orkambi), and care was taken to match the untreated CF subjects by genotypic class of mutations (I-II) and lung function. Thrombin-stimulated calcium entry was higher in platelets obtained from CF subjects not on Orkambi compared with healthy control platelets (Figure 5C, white bars). CFTR inh -172 increased calcium entry in control but not CF platelets, and calcium entry was reduced in both groups by TRPC6 inhibition (Figure 5C). CF platelets from subjects not on Orkambi were also more activated as measured by $\mathrm{CD} 62 \mathrm{P}$ and activated glycoprotein IIb/IIIa (PAC-1) after thrombin compared with controls (Figure 5, D and E, white bars). CFTR $_{\text {inh }}-172$ increased platelet activation in control and CF subjects on Orkambi treatment, but not in CF subjects not receiving Orkambi (Figure 5, D and E). TRPC6 inhibition reduced platelet activation in all groups (Figure $5, \mathrm{D}$ and E). Incubation with SK alone was no different than the CFTR $_{\text {inh }}-172$ + SK group (data not shown). To test for the possible washout of CFTR modulators during the in vitro platelet assay (in CF subjects treated with tezacaftor/ivacaftor), we added back tezacaftor/ivacaftor during platelet incubation, but the results were unchanged (Supplemental Figure 4).

TRPC6 deletion improves lung injury in CF mice. The TRPC6dependency of our in vitro findings of increased calcium entry and activation in CF platelets was tested in the LPS model. Since a specific TRPC6 inhibitor does not exist, we tested CFTR and TRPC6 double-knockout mice. In these littermate experiments, TRPC6 deletion in $\mathrm{CFTR}^{-/-}$mice effectively reversed LPS-induced hyperinflammation, lung permeability, platelet activation, and NET formation (Figure 6, A-F). In the PAO1 model, TRPC6 deletion similarly protected $\mathrm{CFTR}^{--}$mice from hyperinflammation and lung permeability, and also restored bacterial clearance (Figure 6, G-J).

\section{Discussion}

Emerging data support the involvement of hematopoietic cell dysfunction in propagating CF inflammation. The paradoxical hallmark of the CF lung - chronic, acute (neutrophilic) inflammation, including NET formation (49) - has previously been attributed to a downstream effect of pathologic CF mucus colonized with bacteria, but principal defects in CF immune cells may drive this phenotype $(12,13,17)$. We now report on a primary defect in CF platelets as an upstream trigger for neutrophilic inflammation and NET formation in CF lung disease (Figure 7). These findings reframe our conceptualization of CF lung disease to include pathogenic activation of platelets that accompanies the lung epithelial and submucosal gland defects.

Although several animal models of CF have been developed, no single animal model reproduces all of the human features of both acute and chronic lung disease in CF. CFTR-deficient mice do not develop structural lung disease, yet the genetic tools are available in mice for cell-specific interrogation of CFTR function. In addition, CF lung disease is notably a persistent acute inflammation with a neutrophilic predominance, a physiological process for which mouse models are an established tool. Here, we observed that mice with global CFTR deletion have strikingly increased neutrophilic inflammation, NET formation, lung barrier disruption, and impaired bacterial clearance. Furthermore, these mice have increased platelet activation, also observed in human CF disease $(30,50)$, which is a key driver of neutrophilic inflammation and NET formation (38).

We confirmed a previous report (21) that platelets contain CFTR. Platelets that lack functional CFTR channels exhibit increased 
A

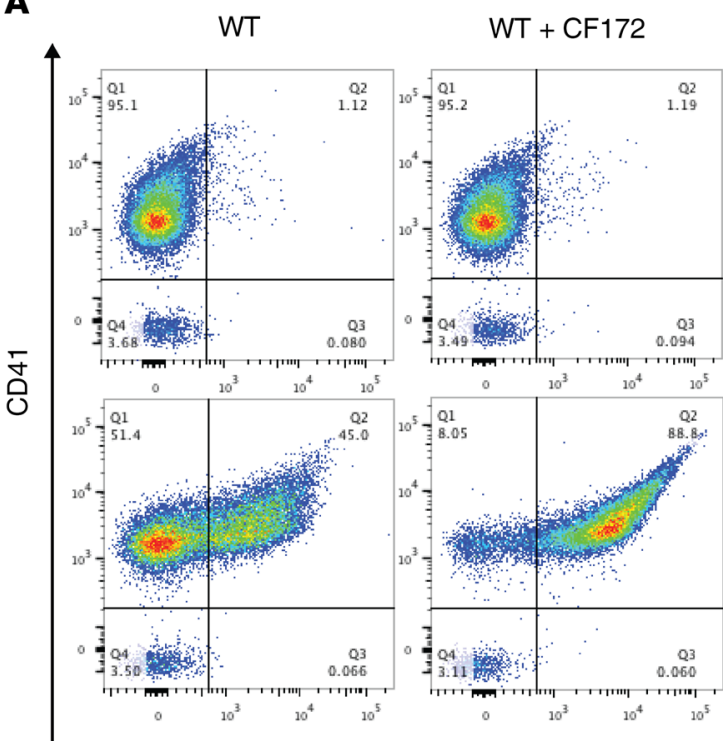

CFTR $C F T R+C F 172$
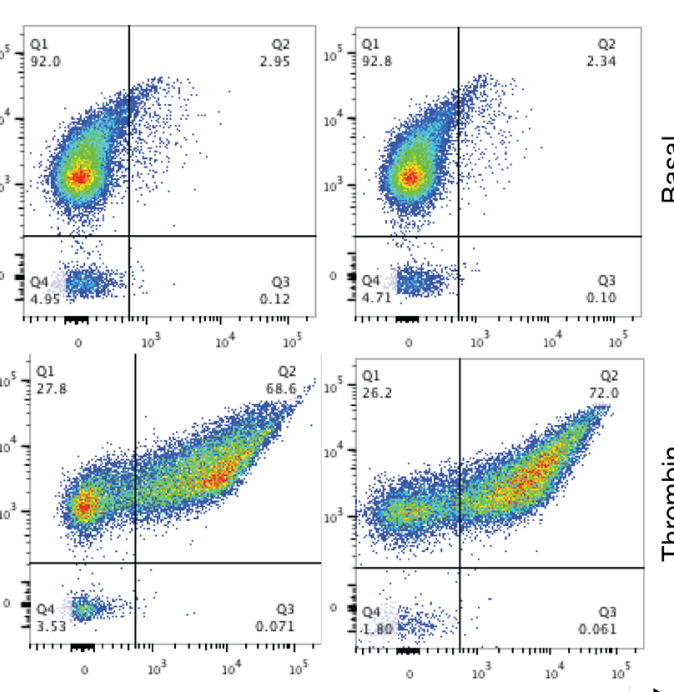

B

CD62P

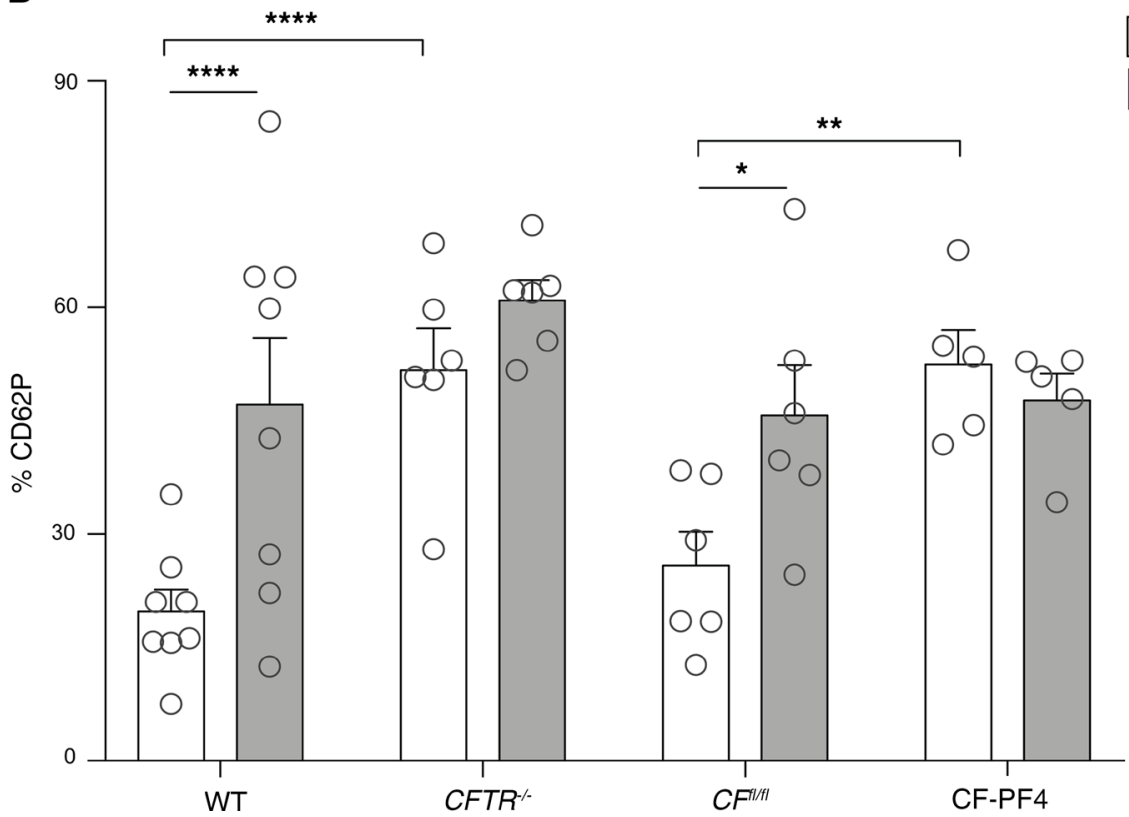

Figure 3. Effects of CFTR inhibition or deletion on thrombin-induced platelet activation. (A) Representative gating scheme of platelet activation (CD41+ events) quantifying CD62P expression with or without thrombin stimulation in WT or CFTR $^{-/-}$platelets with or without CFTRinh-172 (CF172). (B) CD62P expression in platelets from $\mathrm{WT}, \mathrm{CFTR}^{-1-}, \mathrm{CF}^{\mathrm{fl} / \mathrm{fl}}$, and CF-PF4 mice with or without thrombin stimulation plus vehicle or CFTRinh-172 (CF172). Data are mean \pm SEM of 5 to 8 mice. Data were analyzed by 2-way ANOVA. ${ }^{*} P \leq 0.05$; ${ }^{* *} P \leq 0.01$; ${ }^{* * *} P \leq 0.0001$.

calcium entry and are hyperactivated after agonist challenge. Animals with conditional deletion of CFTR in platelets phenocopy the increased lung inflammation and injury seen in $\mathrm{CFTR}^{-/}$mice. Conversely, conditional deletion in myeloid cells or neutrophils alone did not result in hyperinflammatory lung injury in these animal models. Previous work showed an attenuation of lung hyperinflammation after transplanting WT bone marrow into irradiated $\mathrm{CFTR}^{-/}$mice (17), perhaps because of the restoration of CFTR function in megakaryocytes and platelets. Our findings support that platelet activation, resulting from CFTR-mediated augmentation of TRPC 6 activity and calcium entry, is an upstream driver of CF lung inflammation. Importantly, animals with platelet-specific CFTR deletion have intact CFTR in the lung epithelium, which underscores the importance of understanding inflammatory pathway defects in CF lung disease. Deletion of CFTR in platelets led to excessive yet ineffective inflammation, as characterized by defective bacterial clearance even in the presence of increased NETs. This scenario aligns with human CF disease, in which patients exhibit abundant neutrophilic inflammation and NETs in the lungs, yet cannot sterilize their airways.

TRPC6 is 1 of 7 mammalian homologous channels in the TRPC family (51). We found that pharmacologic inhibition or genetic deletion of TRPC6 decreases platelet activation, and reciprocally, calcium entry is increased by pharmacologic or genetic inhibition of CFTR. Our results are also consistent with studies in human airway epithelial cells that showed TRPC6 and CFTR exist in a multiprotein complex with reciprocal functionality (34). In CHO cells, CFTR activation by forskolin downregulated OAG-dependent $\mathrm{Ca}^{2+}$ entry through TRPC6, and in CF cells with the G551D mutation, restoration of CFTR activity by ivacaftor downregulated OAG-dependent $\mathrm{Ca}^{2+}$ entry through TRPC6 (52). This finding suggests that cells with dysfunctional CFTR have aberrant increased TRPC6 activity. 

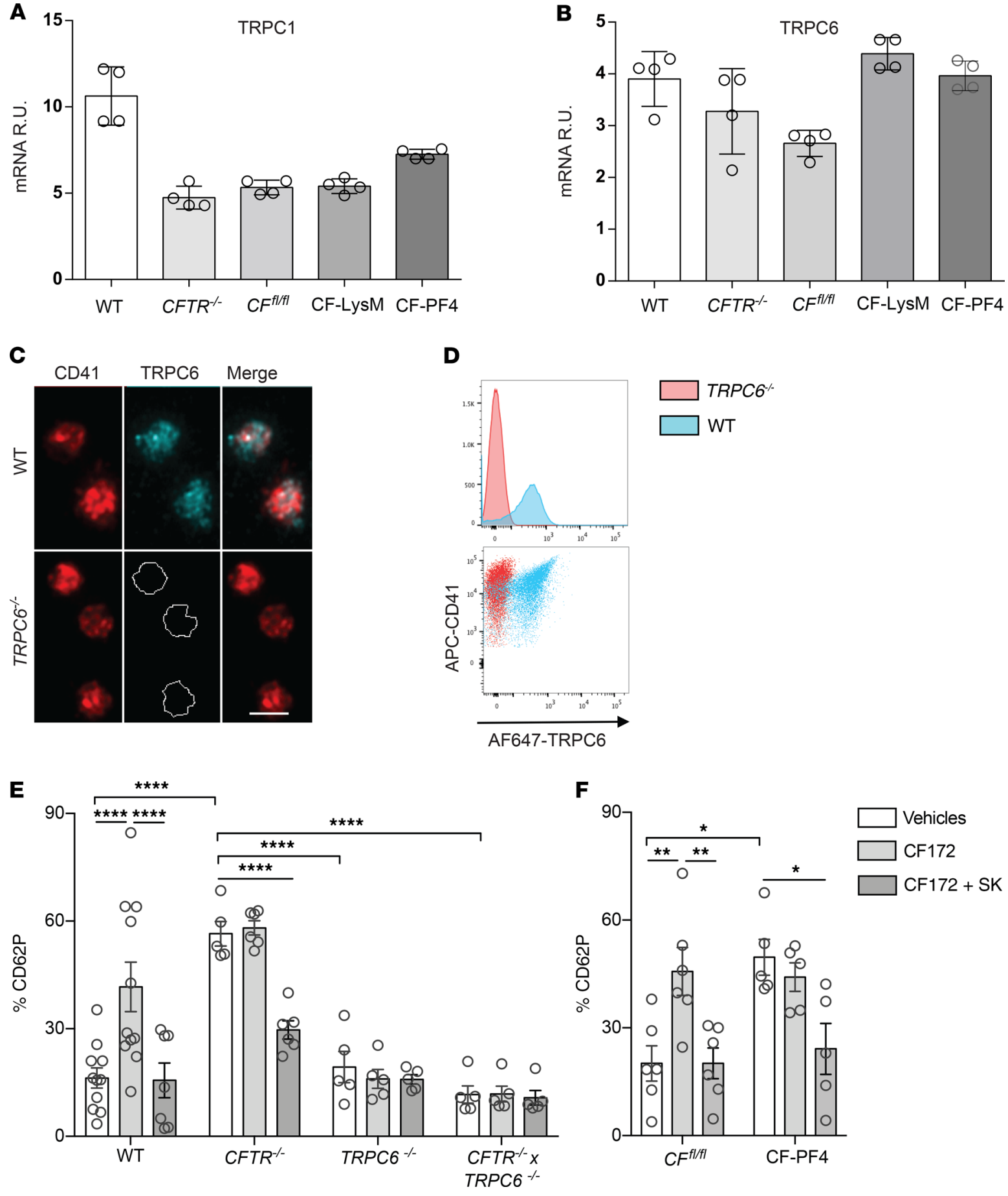

Figure 4. Characterization of TRPC6 in platelets. (A, B) mRNA expression of TRPC isoforms TRPC1 and TRPC6 in platelets from WT, CFTR ${ }^{-/-}, \mathrm{CF}^{f / / f 1}$, CF-LysM, and CF-PF4 mice. TRPC isoforms 2, 3, 4, 5, and 7 were undetectable (not shown). (C) Immunofluorescence staining and (D) flow cytometry analysis of CD41 (red) and TRPC6 (blue) in platelets from WT and TRPC6 ${ }^{-1-}$ mice (representative of 3 independent experiments). Scale bar: $2.5 \mu \mathrm{m}$. (E and F) CD62P expression on platelets from (E) WT, $\mathrm{CFTR}^{-1-}, \mathrm{TRPC6}^{-/-}$, and $C F T R^{-1-} \times \mathrm{TRPC6}^{-/-}$mice, and (F) CF $\mathrm{F}^{\mathrm{fl} / \mathrm{fl}}$ and CF-PF4 mice after thrombin challenge with or without incubation with vehicles, CF172, or CF172 plus SKF-96365 (SK). Data are mean \pm SEM of 5 to 11 animals per group. Data were analyzed by 2 -way ANOVA. ${ }^{*} P \leq 0.05 ;{ }^{* *} P \leq 0.01$; ${ }^{* * *} P \leq 0.0001$.

We extended our animal investigations to human subjects, demonstrating that platelets from healthy controls are hyperactivated after pharmacologic CFTR inhibition, which is reversed by TRPC6 inhibition. Platelets from healthy CF subjects (i.e., not in exacerbation) were hyperactivated compared with healthy con- trols, and this hyperactivation was rescued by TRPC6 inhibition. An interesting observation in the human studies was that CF subjects receiving Orkambi showed increased platelet activation after CFTR inhibition. The significance of these findings are 2-fold: the results suggest that CFTR modulators can restore channel func- 
A
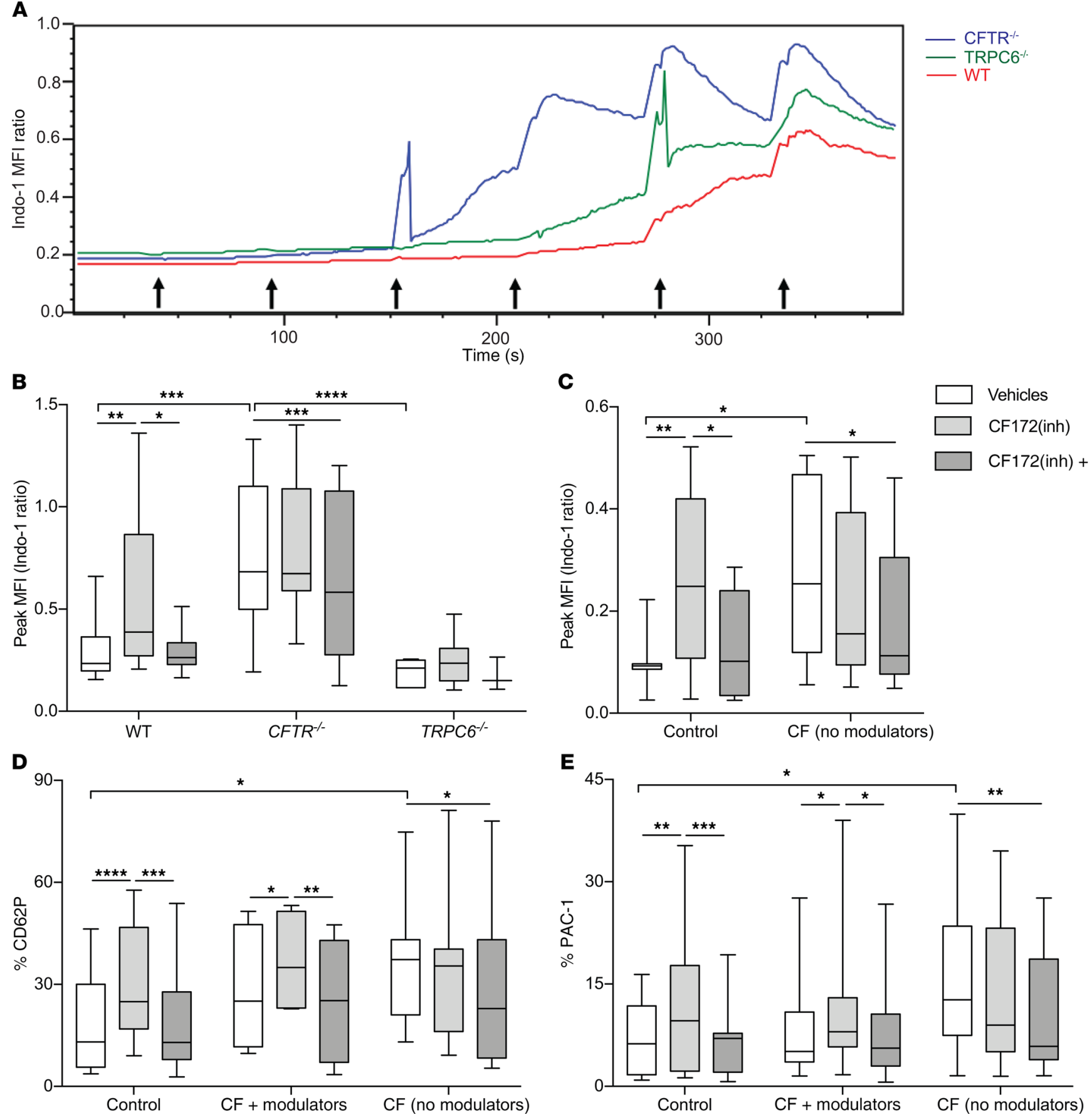

Figure 5. Calcium entry measured by the ratiometric Indo-1 assay in thrombin-stimulated platelets from mice and humans. (A) Kinetic tracings of Indo-1 Violet/Blue MFI measured in platelets isolated from CFTR-/- (blue), TRPC6 ${ }^{-1-}$ (green), and WT (red) mice with $0.125 \mathrm{IU}$ thrombin introduced at 60 -second intervals starting at 30 seconds (black arrows). (B) Peak MFI in WT, CFTR ${ }^{-1-}$, and TRPC6 $6^{-1-}$ platelets incubated with vehicles, CF172, or CF172 plus SK. Data are mean \pm SEM of 7 to 11 animals per group. (C) Peak MFI measured in platelets isolated from healthy human and CF subjects not on modulators incubated with vehicles, CF172, or CF172 plus SK. (D) CD62P and (E) PAC-1 expression in platelets from human controls, CF platelets plus modulators (lumacaftor/ivacaftor), and CF platelets not treated with modulators (no modulators). Data are presented as minimum-to-maximum whiskers and box plots showing the median and interquartile ranges. (C-E) $n=6-12$ subjects per group. Data in B-E were analyzed by 2 -way ANOVA. ${ }^{*} P \leq 0.05 ;{ }^{* *} P \leq 0.01 ;{ }^{* * *} P \leq 0.001 ;{ }^{* * * *} P \leq 0.0001$.

tion in a nonepithelial cell line, and we demonstrate the potential of platelets as a bioassay to assess CFTR modulator efficacy and to facilitate drug development. Theratyping, or the practice of matching medications with mutations, is increasingly important in optimizing which CFTR modulators are efficacious for patients with CF, since epigenetics and unknown factors also determine the severity of phenotype. Invoking a platelet assay to complement existing theratyping analyses, which require epithelial biopsies or brushings, would be a noninvasive method to serially track medication response. 
A

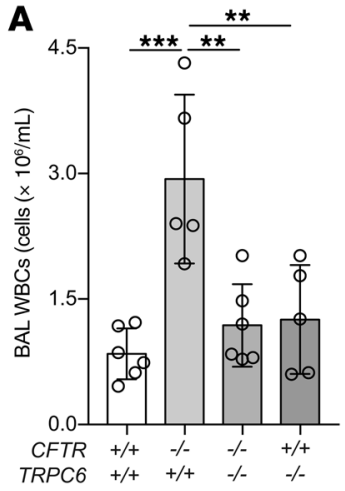

$\stackrel{\infty}{a}$

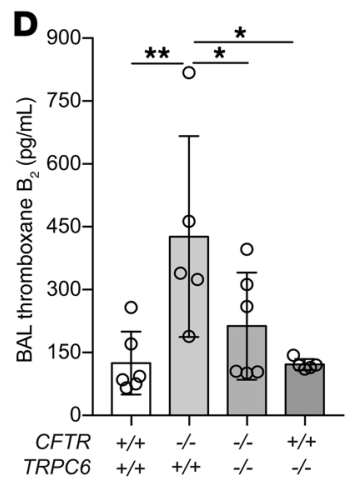

G

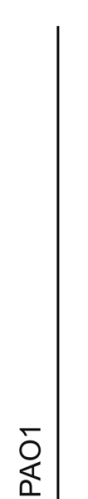

B
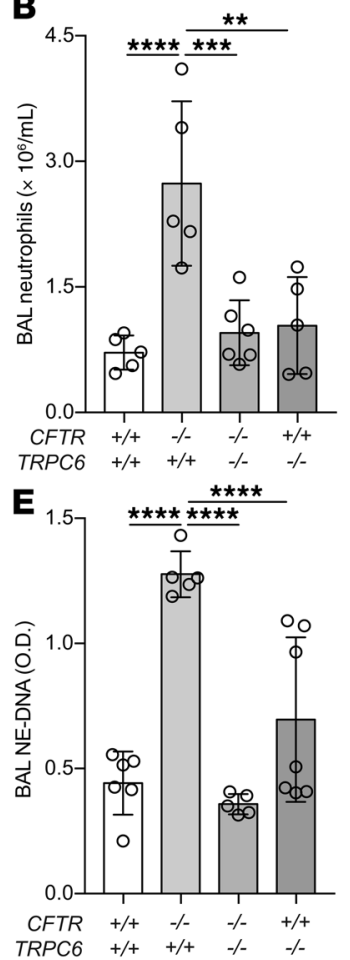

H

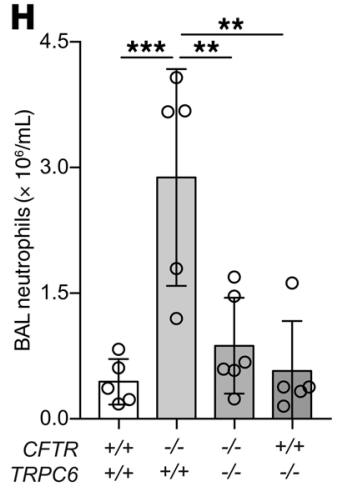

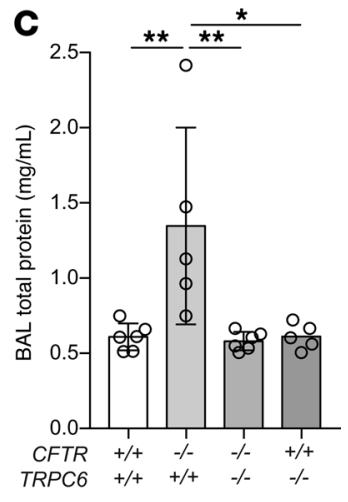
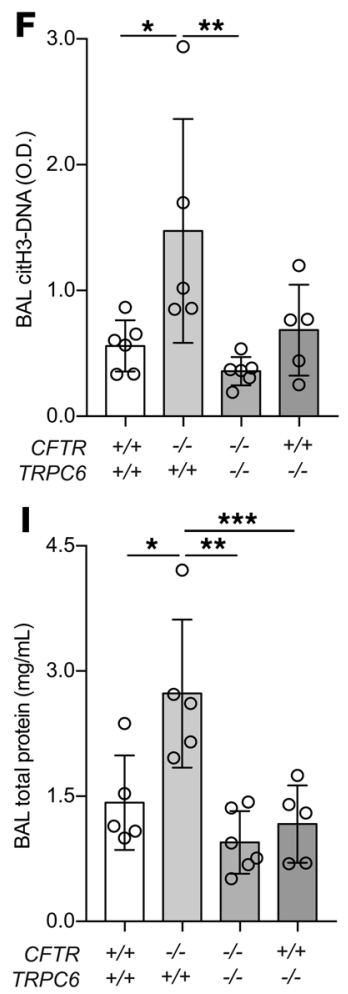

Figure 6. Lung injury measurements in CFTR and TRPC6 mutant mice after intratracheal LPS or PA01. (A) BAL WBCs, (B) neutrophils, (C) total protein, (D) thromboxane $B_{2}$ (E) NETs (NE-DNA ELISA), and (F) NETs (citH3-DNA ELISA) in CFTR $\times$ TRPC6 mutant mice (genotypes indicated in $x$ axis label). Data are mean \pm SEM of 5 to 6 animals per group. Data were analyzed by 2 -way ANOVA. ${ }^{*} P \leq 0.05 ;{ }^{* *} P \leq$ $0.01 ;{ }^{* *} P \leq 0.001 ;{ }^{* * *} P \leq 0.0001$. (G-J) Lung injury and bacterial counts after intratracheal PA01. (C) BAL WBCs, (H) neutrophils, (I) total protein, and (J) lung colonies in CFTR and $T R P C 6$ mutant mice. Data are mean $\pm \mathrm{SEM}$ of 5 to 6 animals per group. Data were analyzed by 2 -way ANOVA. ${ }^{*} P \leq 0.05 ;{ }^{* *} P \leq 0.01 ;{ }^{* *} P$ $\leq 0.001 ;{ }^{* * *} P \leq 0.0001$

calcium entry in CF platelets by targeting TRPC6 is a potentially novel approach, which complements current therapies and provides a new genotype-agnostic treatment. The reversal of lung hyperinflammation and injury we observed in CFTR and TRPC6 double knockouts supports such an approach, although future studies using platelet-specific TRPC6 deletion will be important, since TRPC6 deficiency in the lung endothelium could independently affect lung inflammatory responses $(54,55)$.

Our studies are associated with the following caveats. Using LPS or Pseudomonas aeruginosa to mimic CF lung inflammation is a reductionist approach. Mouse models also do not inform on the structural sequelae of prolonged lung inflammation. Thus, confirming our findings in other animal models such as the ferret, pig, or rabbit will likely be informative. The majority of our studies used 2 pharmacologic inhibitors, CFTR $_{\text {inh }}-172$ and SKF-96365, the latter of which selectively inhibits TRPC3 and TRPC6; however, qPCR studies indicated that platelets do not express TRPC3. Finally, we included a heterogeneous group of $\mathrm{CF}$ mutations in the $\mathrm{CF}$ patients not treated with Orkambi given the scarcity of $\Delta \mathrm{F} 508$ homozygous

Our results are also consistent with older clinical observations that long-term, high-dose ibuprofen slows the progression of CF lung disease particularly in pediatric patients $(31,53)$. We suspect that early inhibition of platelet activation in children with CF decreased platelet-mediated lung inflammation, which partially protected against bronchiectasis and scarring. Anti-platelet therapies in CF are limited by gastrointestinal bleeding and hemoptysis. Inhibiting the abnormal

patients not treated with modulators, but we limited the second mutation to variants within classes I to II.

In conclusion, we define what we believe to be a novel role of CFTR in maintaining platelet homeostasis. In the presence of dysfunctional CFTR, the CF platelet is activated during acute inflammation and drives neutrophilic lung injury. CFTR modulators modestly restore CFTR function on platelets, but improved approaches are needed, such as the direct targeting of TRPC6 in platelets. 


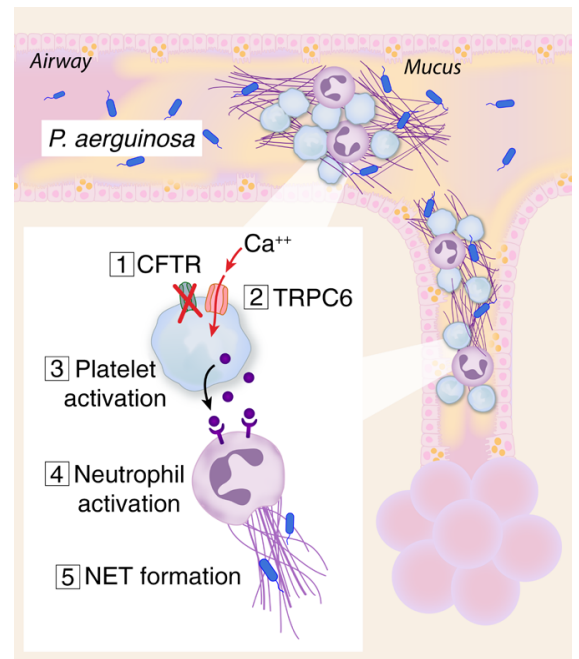

Figure 7. Graphical abstract indicating key events in platelet-induced neutrophilic inflammation in CF.

\section{Methods}

Mice. We used 8- to 12-week-old male mice for the experimental procedures. C57BL/6J and PF4-Cre mice were obtained from The Jackson Laboratory. $C F T R^{-/-}$mice $\left(C f t r^{\text {tmlUnc }}\right)$ and $\mathrm{CF}^{\mathrm{f} / \mathrm{l}}$ mice $\left(C f \mathrm{ft}^{\mathrm{tm} I \mathrm{Cwr}}\right)(40)$ were obtained from the Case Western University Cystic Fibrosis Animal Core. $\mathrm{CFTR}^{-/}$mice express hCFTR protein in the gut under the influence of the rat FABP promoter, and with this gut correction have improved survival (56). TRPC6 ${ }^{-1-}$ mice (B6; 129SvEvTrpc6<Tm1Lbi $>$ ) were provided by FTK (57). LysM-Cre and MRP8-Cre mice were provided by Clifford Lowell (UCSF). Mice were housed and bred under specific pathogen-free conditions at the UCSF Laboratory Animal Research Center and all experiments conformed to ethical principles and guidelines approved by the UCSF Institutional Animal Care and Use Committee.

Lineage-specific deletion of CFTR. Conditional deletion of CFTR was performed using $\mathrm{CF}^{\mathrm{f} / \mathrm{fl}}$ mice crossed with $\mathrm{PF} 4$-Cre mice to delete CFTR in platelets (CF-PF4) (41), LysM-Cre mice to delete CFTR in myeloid cells (CF-LysM) (42), and MRP8-Cre mice to delete CFTR predominantly in neutrophils (CF-MRP8) $(43,58)$. Deletion efficiency was determined using qPCR on isolated cells (see CFTR mRNA analysis by quantitative PCR section, below).

Reagents. The following reagents were used: LPS from Escherichia coli O55:B5 (MilliporeSigma, L4005); CFTR $_{\text {inh }}-172$ (Calbiochem, 219670); SKF-96365 (Abcam, ab130280); BPO27 (synthesized by the lab of ASV, UCSF); human alpha thrombin (Enzyme Research, HT1002a); apyrase (MilliporeSigma, A6535); PGI $_{2}$ (MP Biomedicals, 151969); and Indo-1 (Life Technologies, I1223).

Intratracheal LPS and PAO1 models of lung injury. We used established models of LPS and Pseudomonas aeruginosa-induced lung inflammation and injury $(25,59)$. For the LPS model, mice were anesthetized with ketamine $(80 \mathrm{mg} / \mathrm{kg})$ and xylazine $(12 \mathrm{mg} / \mathrm{kg})$, and LPS was instilled intratracheally (i.t.) at $2.5 \mathrm{mg} / \mathrm{kg}$ under direct visualization (60). At 48 hours, mice were euthanized and blood samples collected by cardiac puncture. A tracheotomy was performed and $1 \mathrm{~mL}$ of PBS +5 mM EDTA was instilled and flushed 3 times to recover the BAL. Quantification of lung injury was carried out using established techniques, including BAL total protein as a measure of lung permeability, and BAL and plasma measurements of total WBCs and differential
(59). BAL analyses, including cell count and leukocyte-platelet aggregate measurements, were done immediately and remaining BAL and plasma were centrifuged, aliquoted, and stored at $-80^{\circ} \mathrm{C}$. BAL WBCs were measured on a Genesis cell counter (Oxford Science). BAL cellular differential was measured with a Cytospin 3 (Thermo Electron, Inc.) and Diff-Quik staining. Total protein measurements in cell-free BAL was measured using a BCA protein assay (Thermo Fisher Scientific).

PAO1 was grown in M9 Minimal Salts media overnight, washed twice in sterile PBS, and resuspended to a concentration of $10^{8} \mathrm{cfu} /$ $\mathrm{mL}$. Stock solutions of PAO1 at the mid-logarithmic growth phase $(2 \times$ $10^{9} \mathrm{cfu} / \mathrm{mL}$ ) were aliquoted and frozen at $-80^{\circ} \mathrm{C}$ in lysogeny broth with glycerol using standard techniques (61). A bacterial slurry at a concentration of $1 \times 10^{6} \mathrm{cfu} /$ mouse was introduced i.t. via direct visualization. In the PAO1 model, lungs were isolated, homogenized in sterile PBS, and the diluted homogenate was spread onto sheep blood agar plates. Colonies were counted at 24 hours.

Flow cytometry. Whole blood was collected into acid citrate dextrose (ACD; MilliporeSigma, C3821). Samples in the presence of Fc $\gamma$ RII/III blocking Ab (2.4G2) were diluted with Tyrode's buffer supplemented with $10 \mathrm{U} / \mathrm{mL}$ heparin (APP), $7 \mathrm{U} / \mathrm{mL}$ Apyrase (MilliporeSigma), and $0.5 \mu \mathrm{M} \mathrm{PGI}$ (MP Biomedicals). eFluor450-CD11b (BD Biosciences, 560456; clone M1/70), PE-Ly6G (BD Biosciences, 551461; clone 1A8), FITC-CD41 (BD Biosciences, 553848; clone MWReg30), APC-CD62P (Invitrogen, 17-0626-82; clone Psel.K02.3), and isotope controls antibodies were used to detect leukocyte and platelet antigens. Samples were analyzed with a LSRII/Fortessa flow cytometer (BD Biosciences). Neutrophils and monocytes were gated by their forward- and side-scatter characteristics, and by their Ly- $6 \mathrm{G}^{+}$/ $\mathrm{CD}_{11 b^{+}}$(neutrophil) or Ly-6G $/ \mathrm{CD} 11 \mathrm{~b}^{+} / \mathrm{Ly}^{-} 6 \mathrm{C}^{+}$(monocyte) expression pattern. NPAs or MPAs were detected by CD41 Ab staining. All data were analyzed using FlowJo software (Tree Star).

CFTR and TRPC6 immunostaining and TRPC6 flow cytometry. For CFTR and TRPC6 immunostaining, platelets from whole blood (WT, $\mathrm{CFTR}^{-/}$, and $\mathrm{TRPC6}^{-/-}$mice) were isolated using centrifugation and then fixed and permeabilized on glass slides. Cells were incubated overnight in PBS-BSA 3\% with or without CFTR (Thermo Fisher Scientific, USA1-935, clone CF3) or TRPC6 (Alomone ACC120, polyclonal) primary antibodies, followed by species-specific Alexa Fluor 647 (Invitrogen, A31571) or Alexa Fluor 488 (Invitrogen, A11006) secondary antibodies, respectively. Anti-CD41 Alexa Fluor 568 (Invitrogen, A11077) was used for platelet staining. Images were captured on a Nikon C1si spectral confocal microscope. For TRPC6 flow cytometry, isolated platelets were incubated with primary and secondary antibodies as described above, and CD $41^{+}$ events were gated for expression of TRPC6.

CFTR Western blot. Gel electrophoresis and immunoblotting was done on isolated human platelets as previously described (62). Briefly, platelet proteins were lysed in RIPA buffer (Thermo Fisher Scientific, 89900), separated on SDS-PAGE (Bio-Rad, 456-1094) and electrophoretically transferred onto nitrocellulose membrane (Bio-Rad, 1662807) before incubations with primary antibodies. The following antibodies were used: human CFTR C-terminus antibody (R\&D Systems, B25031; clone 24-1); $\beta$-Actin antibody (Sigma-Aldrich, A5441; clone AC-15); Rabbit anti-mouse IgG HRP (Abcam, ab6728).

CFTR mRNA analysis by quantitative PCR. Mouse lung was prepared for single-cell analysis (63), and $\mathrm{EpCAM}^{+}$epithelial cells (BioLegend, 118227) were sorted using a BD FACSAria II. Peritoneal 
neutrophils were isolated from $\mathrm{CF}^{\mathrm{f} / \mathrm{l} l}$ and CF-LysM mice using the Cayman Neutrophil Isolation Kit (Cayman Chemicals, 601070) following the manufacturer's instructions, and neutrophil purity was assessed by flow cytometry using Ly6G and CD11b antibodies. Bone marrow megakaryocytes were isolated from $\mathrm{CF}^{\mathrm{fl} / \mathrm{ll}}$ and $\mathrm{CF}-\mathrm{PF} 4$ mice by enrichment with a biotinylated anti-c-Mpl antibody (ImmunoBiological Laboratories, 10403) plus Streptavidin MicroBeads (Miltenyi Biotec, 130-048-101) and isolated with a large cell magnetic column (Miltenyi Biotec, 130-042-202). Megakaryocytes were stained for CD41 (BD Biosciences, 553848) and sorted on a BD FACSAria II with gating parameters based on FSC and CD41+. Megakaryocyte RNA was extracted using a Qiagen RNeasy Micro Kit (Qiagen, 74034). $\mathrm{EpCAM}^{+}$cells and neutrophil RNA were extracted using the Norgen Purification Plus Kit (Norgen Biotek Corp, 47700). cDNA was generated using the High Capacity cDNA Reverse Transcription Kit (Applied Biosystems, 00770980). Multiplex quantitative PCR was performed using custom CFTR-specific TaqMan primers (forward: TGTGGGAAATCCTGTGCTGA; probe-5'6-FAM: CCCTCTGAAGCTTCCAGTTCTCCCA; reverse: AGTACCCGGCATAATCCAAGA) and GAPDH-specific primers (5'-VIC-labeled, 4351309), both from Applied Biosystems. PrimeTime gene expression MasterMix was used for the qPCR (Integrated DNA Technologies, 1055770).

TRPC $m R N A$ analysis by qPCR. Total RNA from platelets was isolated with TRIzol. Reverse transcription was performed (see preceding section) and gene expression of murine TRPC1-TRPC7 was analyzed by qPCR (TaqMan probes, Applied Biosystems). Target gene expression was normalized to housekeeping endogenous control gene $18 \mathrm{~S}$ rRNA (VIC Probe, Applied Biosystems).

CD62P and calcium flux measurements in platelets. Whole blood was collected into ACD by intracardiac puncture (mice) or phlebotomy (humans) and platelets were isolated in Tyrode's buffer supplemented with heparin, apyrase, and $\mathrm{PGI}_{2}$ per protocol (64). Platelets were incubated at a concentration of approximately $3 \times 10^{6}$ per $100 \mu \mathrm{L}$ with $3 \mu \mathrm{M} \mathrm{CFTR}_{\text {inh }}-172$ or $3 \mu \mathrm{M}$ SKF-96365 for 15 minutes before stimulation with 0.125 to $0.5 \mathrm{U} / \mathrm{mL}$ thrombin (standardized throughout a single experiment). APC-CD62P (Invitrogen, 17-062682; clone Psel.K02.3) and FITC-CD41 antibodies were used to gate mouse platelet activation measured on a LSRII/Fortessa flow cytometer (BD Biosciences). In human platelet studies, PE-CD41a (BD Biosciences, 555467; clone HIP8), APC-CD62P (BD Biosciences, 550888; clone AK-4), and FITC-PAC1 (BD Biosciences, 340507; clone PAC-1) were used to gate platelet activation as per above. In selected experiments, human platelets were incubated with $3 \mu \mathrm{M}$ VX-661 (tezacaftor) and $5 \mu \mathrm{M}$ VX-770 (ivacaftor) during the thrombin challenge assay to account for any washout effects from platelet isolation. Calcium entry was detected through a dynamic flow cytometry assay in which isolated platelets were incubated with Indo-1 (a membrane-permeable dye) at room temperature for 40 minutes and stained with APC-CD41 for 15 minutes followed by 2 washes with Tyrode's buffer. Samples were assayed immediately after thrombin stimulation at $485 \mathrm{~nm}$ and $410 \mathrm{~nm}$ wavelengths, which correspond to unbound and calcium-bound Indo-1, respectively (65).
NET and TXB ${ }_{2}$ ELISAs. We have developed custom ELISAs to quantify soluble NET components in plasma and BAL (59). Neutrophil elastase (SCBT M-18 [sc-9521] or G2 [sc-55549]) or citrullinated Histone $\mathrm{H} 3$ antibodies (Abcam, ab5103) were used for capture, and an anti-DNA-HRP conjugate (Cell Death Detection ELISA ${ }^{\text {plus }}$ Kit, Roche) was used as the detection antibody. Thromboxane $\mathrm{B}_{2}$ concentrations in plasma and BAL were determined using an ELISA kit following the manufacturer's instructions (Cayman Chemical).

Platelet aggregation. Platelet aggregation on murine washed platelets was done as previously described (66) on a Lumi-dual aggregometer (Chronolog). The following platelet agonists were tested: collagen type 1 (ABP, ABP-Col-T1) was used at 1 or $2 \mu \mathrm{g} / \mathrm{mL}$; U46619 (MilliporeSigma, 538944) was used at $0.25 \mu \mathrm{M}$; murine thrombin (Sigma-Aldrich, 605157) was used at 0.05 or $0.1 \mathrm{IU} / \mathrm{mL}$.

Tail bleeding time assay. Tail bleeding time on $\mathrm{CF}^{\mathrm{fl} / \mathrm{fl}}$ and CF-PF4 mice was done as previously described (66). Briefly, mice were anesthetized i.p. with ketamine $(25 \mathrm{mg} / \mathrm{kg})$ and xylazine $(10 \mathrm{mg} / \mathrm{kg})$ and placed on a warming blanket. A sterile scalpel was used to transect the tail at a distance of $3 \mathrm{~mm}$ from the tip. A stopwatch was used to monitoring bleeding, and blood drops were removed every 15 seconds with filter paper until bleeding stopped.

Statistics. All in vivo and in vitro experiments were repeated a minimum of 3 independent times. Results are reported as mean \pm SEM. To determine significance, 2-tailed Student's $t$ test and 1-way and 2-way ANOVA tests were used as appropriate (GraphPad PRISM version 7.0). $P$ values of less than or equal to 0.05 were deemed to be significant. Human platelet data are presented as minimum-to-maximum whisker and box plots showing the median and interquartile ranges.

Study approval. All animal experiments were approved by the Institutional Animal Care and Use Committee at the University of California, San Francisco (UCSF). All human subjects were enrolled in a protocol approved by the UCSF Committee for Human Research (IRB).

\section{Author contributions}

GOM, MAY, FTK, PMH, ASV, and MRL designed research studies. GOM, MAY, EL, JJT, BM, SR, CV, KMW, and ZL conducted experiments. GOM, MAY, EL, JJT, BM, SR, CV, KMW, and ZL acquired data. GOM, MAY, EL, JJT, BM, CV, and MRL analyzed data. MAY, NK, DD, MEK, and MRL recruited human subjects. GOM, MAY, and MRL wrote the manuscript. Authorship order for co-first authors was determined based on the relative contributions of these individuals.

\section{Acknowledgments}

This work was supported by a Cystic Fibrosis Foundation 3rd Year Clinical Fellowship and LeRoy Matthews Physician-Scientist Award (MAY), a Cystic Fibrosis Foundation Research Development Program Pilot and Feasibility Award (MRL), and NIH grants R01 HL107386 and R01 AI125445 (MRL) and NIH P30 DK72517 (ASV).

Address correspondence to: Mark R. Looney, 513 Parnassus Avenue, HSE 1355A, San Francisco, California 94143-0130, USA. Phone: 415.476.9563; Email: mark.looney@ucsf.edu.

\footnotetext{
1. Cystic Fibrosis Foundation. Patient Registry Annual Data Report 2017. Bethesda, Maryland, USA: Cystic Fibrosis Foundation; 2017.

2. Elborn JS. Cystic fibrosis. Lancet.
}

2016;388(10059):2519-2531.

3. Riordan JR, et al. Identification of the cystic fibrosis gene: cloning and characterization of complementary DNA. Science. 1989;245(4922):1066-1073.
4. Fuchs $\mathrm{HJ}$, et al. Effect of aerosolized recombinant human DNase on exacerbations of respiratory symptoms and on pulmonary function in patients with cystic fibrosis. The Pulmozyme Study 
Group. N Engl J Med. 1994;331(10):637-642.

5. Ramsey BW, et al. A CFTR potentiator in patients with cystic fibrosis and the G551D mutation. N Engl JMed. 2011;365(18):1663-1672.

6. Wainwright CE, et al. Lumacaftor-ivacaftor in patients with cystic fibrosis homozygous for Phe508del CFTR. N Engl JMed. 2015;373(3):220-231.

7. Taylor-Cousar JL, et al. Tezacaftor-ivacaftor in patients with cystic fibrosis homozygous for Phe508del. NEngl J Med. 2017;377(21):2013-2023.

8. Rowe SM, et al. Tezacaftor-ivacaftor in residualfunction heterozygotes with cystic fibrosis. N Engl J Med. 2017;377(21):2024-2035.

9. Hurley MN, McKeever TM, Prayle AP, Fogarty AW, Smyth AR. Rate of improvement of CF life expectancy exceeds that of general population--observational death registration study. J Cyst Fibros. 2014;13(4):410-415.

10. Matsui $\mathrm{H}$, et al. Evidence for periciliary liquid layer depletion, not abnormal ion composition, in the pathogenesis of cystic fibrosis airways disease. Cell. 1998;95(7):1005-1015.

11. Sly PD, et al. Risk factors for bronchiectasis in children with cystic fibrosis. $N$ Engl J Med. 2013;368(21):1963-1970.

12. Rosen $\mathrm{BH}$, et al. Infection is not required for mucoinflammatory lung disease in CFTR-knockout ferrets. Am J Respir Crit Care Med. 2018;197(10):1308-1318.

13. Keiser NW, et al. Defective innate immunity and hyperinflammation in newborn cystic fibrosis transmembrane conductance regulator-knockout ferret lungs. Am J Respir Cell Mol Biol. 2015;52(6):683-694.

14. Rogers CS, et al. Disruption of the CFTR gene produces a model of cystic fibrosis in newborn pigs. Science. 2008;321(5897):1837-1841.

15. Stoltz DA, et al. Cystic fibrosis pigs develop lung disease and exhibit defective bacterial eradication at birth. Sci Transl Med. 2010;2(29):29ra31.

16. Su X, Looney MR, Su HE, Lee JW, Song Y, Matthay MA. Role of CFTR expressed by neutrophils in modulating acute lung inflammation and injury in mice. Inflamm Res. 2011;60(7):619-632.

17. Bruscia EM, et al. Macrophages directly contribute to the exaggerated inflammatory response in cystic fibrosis transmembrane conductance regulator-/- mice. Am J Respir Cell Mol Biol. 2009;40(3):295-304.

18. Painter RG, Marrero L, Lombard GA, Valentine VG, Nauseef WM, Wang G. CFTR-mediated halide transport in phagosomes of human neutrophils. J Leukoc Biol. 2010;87(5):933-942.

19. Painter RG, et al. CFTR Expression in human neutrophils and the phagolysosomal chlorination defect in cystic fibrosis. Biochemistry. 2006;45(34):10260-10269.

20. Di A, et al. CFTR regulates phagosome acidification in macrophages and alters bactericidal activity. Nat Cell Biol. 2006;8(9):933-944.

21. Mattoscio D, et al. Cystic fibrosis transmembrane conductance regulator (CFTR) expression in human platelets: impact on mediators and mechanisms of the inflammatory response. FASEB J. 2010;24(10):3970-3980.

22. Bruscia EM, et al. Abnormal trafficking and degradation of TLR4 underlie the elevated inflam- matory response in cystic fibrosis. Jimmunol. 2011;186(12):6990-6998

23. Deppermann C, Kubes P. Start a fire, kill the bug: The role of platelets in inflammation and infection. Innate Immun. 2018;24(6):335-348.

24. Looney MR, Nguyen JX, Hu Y, Van Ziffle JA, Lowell CA, Matthay MA. Platelet depletion and aspirin treatment protect mice in a two-event model of transfusion-related acute lung injury. J Clin Invest. 2009;119(11):3450-3461.

25. Ortiz-Muñoz G, Mallavia B, Bins A, Headley M, Krummel MF, Looney MR. Aspirin-triggered 15-epi-lipoxin A4 regulates neutrophil-platelet aggregation and attenuates acute lung injury in mice. Blood. 2014;124(17):2625-2634.

26. Rossaint J, et al. Directed transport of neutrophil-derived extracellular vesicles enables platelet-mediated innate immune response. Nat Commun. 2016;7:13464.

27. Bain W, et al. Platelets inhibit apoptotic lung epithelial cell death and protect mice against infection-induced lung injury. Blood $A d v$. 2019;3(3):432-445.

28. Amison RT, et al. Platelet depletion impairs host defense to pulmonary infection with pseudomonas aeruginosa in mice. Am J Respir Cell Mol Biol. 2018;58(3):331-340.

29. Mahaut-Smith MP. Chloride channels in human platelets: evidence for activation by internal calcium. J Membr Biol. 1990;118(1):69-75.

30. O'Sullivan BP, et al. Platelet activation in cystic fibrosis. Blood. 2005;105(12):4635-4641.

31. Konstan MW, Byard PJ, Hoppel CL, Davis PB. Effect of high-dose ibuprofen in patients with cys tic fibrosis. N Engl J Med. 1995;332(13):848-854.

32. Markworth JF, et al. Human inflammatory and resolving lipid mediator responses to resistance exercise and ibuprofen treatment. Am J Physiol Regul Integr Comp Physiol. 2013;305(11):R1281-R1296.

33. Zhao C, et al. Important role of platelets in modulating endotoxin-induced lung inflammation in CFTR-deficient mice. PLOS ONE. 2013;8(12):e82683.

34. Antigny F, et al. Transient receptor potential canonical channel 6 links Ca2+ mishandling to cystic fibrosis transmembrane conductance regulator channel dysfunction in cystic fibrosis. Am J Respir Cell Mol Biol. 2011;44(1):83-90.

35. Albarran L, et al. TRPC6 participates in the regulation of cytosolic basal calcium concentration in murine resting platelets. Biochim Biophys Acta. 2014;1843(4):789-796.

36. Vemana HP, Karim ZA, Conlon C, Khasawneh FT. A critical role for the transient receptor potential channel type 6 in human platelet activation. PLOS ONE. 2015;10(4):e0125764.

37. Clark SR, et al. Platelet TLR4 activates neutrophil extracellular traps to ensnare bacteria in septic blood. Nat Med. 2007;13(4):463-469.

38. Caudrillier A, et al. Platelets induce neutrophil extracellular traps in transfusion-related acute lung injury. J Clin Invest. 2012;122(7):2661-2671.

39. Kraemer BF, et al. Novel anti-bacterial activities of $\beta$-defensin 1 in human platelets: suppression of pathogen growth and signaling of neutrophil extracellular trap formation. PLoS Pathog. 2011;7(11):e1002355.
40. Hodges CA, Cotton CU, Palmert MR, Drumm ML. Generation of a conditional null allele for Cftr in mice. Genesis. 2008;46(10):546-552.

41. Tiedt R, Schomber T, Hao-Shen H, Skoda RC. Pf4-Cre transgenic mice allow the generation of lineage-restricted gene knockouts for studying megakaryocyte and platelet function in vivo. Blood. 2007;109(4):1503-1506.

42. Clausen BE, Burkhardt C, Reith W, Renkawitz $\mathrm{R}$, Förster I. Conditional gene targeting in macrophages and granulocytes using LysMcre mice. Transgenic Res. 1999;8(4):265-277.

43. Passegué E, Wagner EF, Weissman IL. JunB deficiency leads to a myeloproliferative disorder arising from hematopoietic stem cells. Cell. 2004;119(3):431-443.

44. Ma T, et al. Thiazolidinone CFTR inhibitor identified by high-throughput screening blocks cholera toxin-induced intestinal fluid secretion. JClin Invest. 2002;110(11):1651-1658.

45. Snyder DS, Tradtrantip L, Yao C, Kurth MJ, Verkman AS. Potent, metabolically stable benzopyrimido-pyrrolo-oxazine-dione (BPO) CFTR inhibitors for polycystic kidney disease. JMed Chem. 2011;54(15):5468-5477.

46. Hassock SR, Zhu MX, Trost C, Flockerzi V, Authi KS. Expression and role of TRPC proteins in human platelets: evidence that TRPC6 forms the store-independent calcium entry channel. Blood. 2002;100(8):2801-2811.

47. Varga-Szabo D, et al. Store-operated $\mathrm{Ca}(2+)$ entry in platelets occurs independently of transient receptor potential (TRP) C1. Pflugers Arch. 2008;457(2):377-387.

48. Singh A, Hildebrand ME, Garcia E, Snutch TP. The transient receptor potential channel antagonist SKF96365 is a potent blocker of low-voltage-activated T-type calcium channels. Br J Pharmacol. 2010;160(6):1464-1475.

49. Gray RD, et al. Delayed neutrophil apoptosis enhances NET formation in cystic fibrosis. Thorax. 2018;73(2):134-144.

50. O'Sullivan BP, Michelson AD. The inflammatory role of platelets in cystic fibrosis. Am J Respir Crit Care Med. 2006;173(5):483-490.

51. Feske S, Wulff H, Skolnik EY. Ion channels in innate and adaptive immunity. Annu Rev Immunol. 2015;33:291-353.

52. Vachel L, Norez C, Becq F, Vandebrouck C. Effect of VX-770 (ivacaftor) and OAG on Ca2+ influx and CFTR activity in G551D and F508del-CFTR expressing cells. J Cyst Fibros. 2013;12(6):584-591.

53. Konstan MW, Schluchter MD, Xue W, Davis PB. Clinical use of ibuprofen is associated with slower FEV1 decline in children with cystic fibrosis. Am J Respir Crit Care Med. 2007;176(11):1084-1089.

54. Tauseef M, et al. TLR4 activation of TRPC6dependent calcium signaling mediates endotoxininduced lung vascular permeability and inflammation. JExp Med. 2012;209(11):1953-1968.

55. Weber EW, Han F, Tauseef M, Birnbaumer L, Mehta D, Muller WA. TRPC6 is the endothelial calcium channel that regulates leukocyte transendothelial migration during the inflammatory response. JExp Med. 2015;212(11):1883-1899.

56. Zhou L, Dey CR, Wert SE, DuVall MD, Frizzell RA, Whitsett JA. Correction of lethal intestinal defect 
in a mouse model of cystic fibrosis by human CFTR. Science. 1994;266(5191):1705-1708.

57. Paez Espinosa EV, Murad JP, Ting HJ, Khasawneh FT. Mouse transient receptor potential channel 6: role in hemostasis and thrombogenesis. Biochem Biophys Res Commun. 2012;417(2):853-856.

58. Van Ziffle JA, Lowell CA. Neutrophil-specific deletion of Syk kinase results in reduced host defense to bacterial infection. Blood. 2009;114(23):4871-4882.

59. Lefrançais E, Mallavia B, Zhuo H, Calfee CS, Looney MR. Maladaptive role of neutrophil extracellular traps in pathogen-induced lung injury. JCI Insight. 2018;3(3):98178.

60. Ortiz-Muñoz G, Looney MR. Non-invasive intratracheal instillation in mice. Bio Protoc. 2015;5(12):e1504.

61. Tran CS, et al. The pseudomonas aeruginosa type III translocon is required for biofilm formation at the epithelial barrier. PLoS Pathog. 2014;10(11):e1004479.

62. Valet C, et al. Essential role of class II PI3K$\mathrm{C} 2 \alpha$ in platelet membrane morphology. Blood. 2015;126(9):1128-1137.

63. Lefrançais E, et al. The lung is a site of platelet biogenesis and a reservoir for haematopoietic progenitors. Nature. 2017;544(7648):105-109.

64. Krueger LA, Barnard MR, Frelinger AL, Furman MI, Michelson AD. Immunophenotypic analysis of platelets. Curr Protoc Cytom. 2002; Chapter 6: 6.10.1-6.10.17.

65. Jennings LK, Dockter ME, Wall CD, Fox CF, Kennedy DM. Calcium mobilization in human platelets using indo-1 and flow cytometry. Blood. 1989;74(8):2674-2680.

66. Valet C, et al. A dual role for the class III PI3K, Vps34, in platelet production and thrombus growth. Blood. 2017;130(18):2032-2042. 